\title{
Slutsky Revisited: A New Decomposition of the Price Effect
}

\author{
Kazuyuki Sasakura ${ }^{1}$
}

Received: 26 February 2016 / Accepted: 26 April 2016 / Published online: 9 May 2016

(C) Società Italiana degli Economisti (Italian Economic Association) 2016

\begin{abstract}
It is only the Slutsky equation that has been universally used to examine how the demand for a good responds to variations in its own price. This paper proposes an alternative to the Slutsky equation. It decomposes such a price effect into the "ratio effect" and the "unit-elasticity effect". The "ratio effect" is positive (negative) if the expenditure spent on a good under consideration increases (decreases) when its own price rises, and it can be divided further into the familiar substitution effect and the "transfer effect" which reflects the income effect of other goods. The "unit-elasticity effect," which is always negative, stands for unitary price elasticity of demand. It is also shown that the new method can be used for the analysis of the cross-price effect with and without initial endowments. The Slutsky equation and the new one are "complements", but graphical representations as well as examples of the applications reveal that the latter is much easier to understand intuitively.
\end{abstract}

Keywords The Slutsky equation · Price effect · Ratio effect · Unit-elasticity effect

\section{JEL Classification D11}

\footnotetext{
I express my deepest gratitude to Professor Masamichi Kawano, Kwansei Gakuin University, and participants at the 2010 fall meeting of the Japanese Economic Association for their useful comments. This paper has also greatly improved due to detailed comments from an anonymous referee and the editor of this journal. Needless to say, the usual caveats apply.

$凶$ Kazuyuki Sasakura sasakura@waseda.jp

1 Faculty of Political Science and Economics, Waseda University, 1-6-1 Nishiwaseda, Shinjuku-ku, Tokyo 169-8050, Japan
} 


\section{Introduction}

The very first mission of demand theory was, is, and will be to analytically answer the question of how the demand for a good responds to variations in its own price (the price effect). Since Pareto (1892) discovered, and both Slutsky (1915) and Hicks and Allen (1934a,b) gave an elegant formulation, it is only the Slutsky equation that has been universally used for such an analysis. The Slutsky equation teaches us, quite correctly, that the price effect can be decomposed into the substitution effect and the income effect (the Slutsky decomposition). It has been the most fundamental tool not only for pure demand theory but also for wide applications, microeconomic or macroeconomic. It is no exaggeration to say that without it economists could have been only half through their works. The contribution of the Slutsky equation to economics is literally immeasurable. ${ }^{1}$

On the other hand, current workhorses in demand theory are demand functions derived from the Cobb-Douglas utility function and the CES utility function which is a generalized version of the Cobb-Douglas. It is known that in such cases the income effect is always negative. In other words all goods are normal ones. Since the substitution effect is negative in general, the Slutsky equation repeats a matter of course in a sense, i.e., a negative substitution effect and a negative income effect lead to a negative price effect. This statement itself is very helpful and no one can deny it. But now it may be too ordinary to grab academic researchers. Then, is there an alternative to it?

In this paper I propose another way to decompose the price effect. According to it, the price effect is composed of the "ratio effect" and the "unit-elasticity effect." The "ratio effect" is positive (negative) if the expenditure spent on a good under consideration increases (decreases) when its own price goes up, and it can be divided further into the familiar substitution effect and the "transfer effect" which reflects the income effect of other goods. The "unit-elasticity effect" indicates how the demand for the good changes in response to a rise in its own price if the expenditure for the good remains unchanged, i.e., if its price elasticity of demand is unity. The "unit-elasticity effect" thus defined is always negative.

The usefulness of the new decomposition is the most understandable in the CobbDouglas and the CES cases. Particularly in the Cobb-Douglas case, as will be seen below, the "ratio effect" vanishes because a negative substitution effect and a positive "transfer effect" just cancel out. The new decomposition would be applicable to other various problems. For example, not a few economists may be interested in the case where the ratio effect is large enough to govern the price effect, i.e., the case of a Giffen good. It never occurs in the Cobb-Douglas or in the CES case. The new method can also be applied to the analysis of such a good.

This paper is organized as follows. Reviewing the Slutsky equation, Sect. 2 presents a new equation which decomposes the price effect into the ratio effect and the unitelasticity effect. Applications of the two equations to examples often used in economics are given, too. Section 3 explains the new equation graphically for a better understand-

\footnotetext{
${ }^{1}$ For the relationship among Pareto (1892), Slutsky (1915), and Hicks and Allen (1934a, b), see Allen (1936) and Dooley (1983). See also Schultz (1935) for the first empirical application of the Slutsky equation.
} 
ing. The new method is applies to the case of a Giffen good in Sect. 4, the cross-price case in Sect. 5, and a case where a consumer holds initial endowments in Sect. 6. Section 7 concludes the paper.

\section{Comparison of a New Decomposition with the Slutsky}

A consumer under consideration has an ordinal utility function

$$
v=u\left(q_{1}, q_{2}\right)
$$

where $q_{1}$ and $q_{2}$ are the quantity of good 1 and that of good 2 , respectively. ${ }^{2}$ For simplicity both good 1 and good 2 are assumed to be normal except in Sect. 4. As usual, the utility function (1) has the following properties:

$$
u_{1}>0, u_{2}>0
$$

and

$$
\begin{aligned}
|U| & \equiv\left|\begin{array}{ccc}
u_{11} & u_{12} & u_{1} \\
u_{21} & u_{22} & u_{2} \\
u_{1} & u_{2} & 0
\end{array}\right| \\
& =-u_{2}^{2} u_{11}+2 u_{1} u_{2} u_{12}-u_{1}^{2} u_{22}>0
\end{aligned}
$$

where $u_{i}=\partial u / \partial q_{i}$ and $u_{i j}=\partial^{2} u / \partial q_{i} \partial q_{j}, i, j=1,2$. The positivity of bordered Hessian $|U|$ means the decreasing marginal rate of substitution. ${ }^{3}$

The objective of the consumer is to maximize the utility represented by $u\left(q_{1}, q_{2}\right)$ under the budget constraint $p_{1} q_{1}+p_{2} q_{2}=y$ with prices $p_{1}, p_{2}$ of goods 1,2 and income $y$ as given. This utility maximization problem can be written compactly as

$$
\begin{aligned}
\text { Problem I : } & \max _{q_{1}, q_{2}} u\left(q_{1}, q_{2}\right) \\
\text { s.t. } & p_{1} q_{1}+p_{2} q_{2}=y .
\end{aligned}
$$

The solutions, $q_{1}^{*}, q_{2}^{*}$, to this problem, i.e., the demands for goods 1 and 2 , are the function of $p_{1}, p_{2}$, and $y$. Then, the Slutsky equation says that the price effect with respect to good 1 is expressed as the sum of the substitution effect and the income effect as follows:

\footnotetext{
2 The arguments below will proceed around good 1. Good 2 can be regarded as the rest of all goods.

${ }^{3}$ Utility must be ordinal. This is what Slutsky (1915) and Hicks and Allen (1934a, b) emphasized in order to say good-bye to the unrealistic assumption that utility is measurable. Economists should have replaced a cardinal utility with an ordinal one. Nevertheless, the former did not die. Rather, it revived and is now prospering in economic theory such as game theory, expected utility theory, and optimal growth theory. Did they forget the great contribution by Slutsky et al.? For the related discussion of expected utility theory, see Montesano (1985).
} 


$$
\underbrace{\left.\frac{d q_{1}}{d p_{1}}\right|_{p_{2}, y=\text { const }}}_{\text {price effect }}=\underbrace{\left.\frac{d q_{1}}{d p_{1}}\right|_{p_{2}, v=\text { const }}}_{\text {substitution effect }}+\underbrace{\left(-\left.q_{1} \frac{d q_{1}}{d y}\right|_{p_{1}, p_{2}=\text { const }}\right)}_{\text {income effect }},
$$

where

$$
\begin{aligned}
\left.\frac{d q_{1}}{d p_{1}}\right|_{p_{2}, v=\mathrm{const}} & =-\frac{u_{1} u_{2}^{2}}{p_{1}|U|}<0 \text { and }-\left.q_{1} \frac{d q_{1}}{d y}\right|_{p_{1}, p_{2}=\mathrm{const}} \\
& =-q_{1} \frac{u_{1}\left(u_{2} u_{12}-u_{1} u_{22}\right)}{p_{1}|U|}<0,
\end{aligned}
$$

all partial derivatives and $q_{1}$ being evaluated at $\left(q_{1}^{*}, q_{2}^{*}\right)$. The above arguments are too ordinary to need more explanation. Graphical representations of it can easily be found in every textbook of microeconomics.

Here, for concreteness, let us apply the Slutsky equation (2) to two familiar examples of (1). One is the Cobb-Douglas-type utility function

$$
v=A q_{1}^{a} q_{2}^{b}, \quad a>0, b>0
$$

with $A, a$, and $b$ as positive parameters. The Slutsky equation for this utility function becomes

$$
\underbrace{-\frac{a}{a+b} \frac{y}{p_{1}^{2}}}_{\text {price effect }}=\underbrace{-\frac{a b}{(a+b)^{2}} \frac{y}{p_{1}^{2}}}_{\text {substitution effect } \ominus}+\underbrace{\left[-\frac{a^{2}}{(a+b)^{2}} \frac{y}{p_{1}^{2}}\right]}_{\text {income effect } \ominus}<0 .
$$

Good 1 is certainly a normal good. The other example is the CES utility function

$$
v=A\left(a q_{1}^{\frac{\sigma-1}{\sigma}}+b q_{2}^{\frac{\sigma-1}{\sigma}}\right)^{\frac{\sigma}{\sigma-1}}
$$

with $A, a, b$, and $\sigma$ as positive parameters. $\sigma$ is referred to as the elasticity of substitution between good 1 and good $2 .^{4}$ The Slutsky equation in this case is written as

$$
\underbrace{-\frac{1+\sigma c}{(1+c)^{2}} \frac{y}{p_{1}^{2}}}_{\text {price effect }}=\underbrace{-\frac{\sigma c}{(1+c)^{2}} \frac{y}{p_{1}^{2}}}_{\text {substitution effect } \ominus}+\underbrace{\left[-\frac{1}{(1+c)^{2}} \frac{y}{p_{1}^{2}}\right]}_{\text {income effect } \ominus}<0,
$$

where

$$
c=\left(\frac{b}{a}\right)^{\sigma}\left(\frac{p_{1}}{p_{2}}\right)^{\sigma-1}>0 .
$$

\footnotetext{
4 When $\sigma=1$ and $a+b=1$, the CES utility function (5) coincides with the Cobb-Douglas utility function (3). For the proof, see Henderson and Quandt (1980, p. 113).
} 
Again good 1 is a normal good. As seen from these examples, the Slutsky equation goes on justifying the law of demand as long as a good is a normal one.

Now let me propose another decomposition. It is derived from the solutions to the following problem which is obtained simply by rewriting Problem I just a little bit:

$$
\begin{aligned}
& \text { Problem II : } \max _{\theta} u\left(q_{1}, q_{2}\right) \\
& \text { s.t. } q_{1}=\frac{\theta y}{p_{1}}, q_{2}=\frac{(1-\theta) y}{p_{2}}, \quad 0 \leq \theta \leq 1 .
\end{aligned}
$$

$\theta$ is the ratio of income $y$ that goes to the purchase of good $1 .{ }^{5}$ As is apparent, Problem I and Problem II are mathematically equivalent and the solutions to each problem are the same. ${ }^{6}$ The difference is that the optimal quantities, $q_{1}^{*}, q_{2}^{*}$, of goods 1 and 2 are found in Problem I by adjusting those quantities $q_{1}$ and $q_{2}$ directly, whereas in Problem II by controlling the ratio $\theta$ indirectly. It seems trifling. But differentiating $q_{1}=\frac{\theta y}{p_{1}}$ partially with respect to $p_{1}$ gives quite a new equation:

$$
\underbrace{\left.\frac{d q_{1}}{d p_{1}}\right|_{p_{2}, y=\text { const }}}_{\text {price effect }}=\underbrace{\frac{\frac{d \theta}{d p_{1}} y}{p_{1}}}_{\text {ratio effect }}+\underbrace{\left(\left.\frac{d q_{1}}{d p_{1}}\right|_{y, \theta=\text { const }}\right)}_{\text {unit-elasticity effect }},
$$

where

$\frac{\frac{d \theta}{d p_{1}} y}{p_{1}}=-\frac{u_{1}}{p_{1}} \frac{u_{2}^{2}}{|U|}+\frac{q_{1}}{p_{1}} \frac{u_{1} u_{2} u_{12}-u_{2}^{2} u_{11}}{|U|} \gtreqless 0 \quad$ and $\left.\quad \frac{d q_{1}}{d p_{1}}\right|_{y, \theta=\mathrm{const}}=-\frac{q_{1}}{p_{1}}<0$,

all partial derivatives and $q_{1}$ being evaluated at $\left(q_{1}^{*}, q_{2}^{*}\right)$.

According to (7), the price effect can be divided into the two effects which are called for convenience the "ratio" effect and the "unit-elasticity" effect. The implication is very simple. It is this. When price $p_{1}$ of good 1 rises, a change in the demand for it is governed completely by the unit-elasticity effect unless the ratio $\theta$ changes, or in other words if the expenditure spent on it remains unchanged. The magnitude of it can be calculated from the relation $p_{1} q_{1}=\theta y$ with $\theta$ and $y$ as given. Total differentiation of it yields the value of $-q_{1} / p_{1}$ at once. To put it differently, a unit increase in $p_{1}$ decreases income $y$ by $q_{1}$ which in turn implies a decrease in the demand for good 1 by $q_{1} / p_{1}$, ceteris paribus. In a word, the price elasticity of demand is unity. Hence the name "unit-elasticity" effect. 7

Of course, the price effect differs from the unit-elasticity effect when the ratio $\theta$ is affected by variations in $p_{1}$. The "ratio" effect measures the difference. If the ratio changes by $d \theta / d p_{1}$, it means a variation in the expenditure for good 1 by $\left(d \theta / d p_{1}\right) y$

\footnotetext{
5 For other interpretations of $\theta$, see footnotes 18 and 21 below.

6 The conditions for utility maximization are derived from Problem II in "Appendix 1".

7 Note that the statement of this paragraph holds in the n-good case, too. Particularly the way to derive the unit-elasticity effect is always the same.
} 
which in turn leads to a change in the demanded quantity by $\left(d \theta / d p_{1}\right) y / p_{1}$. This is the ratio effect. The calculation of it is not so easy as that of the unit-elasticity effect. Fortunately, however, the value of the price effect is already known in (2). Thus, the quickest way to obtain the ratio effect is to subtract $-q_{1} / p_{1}$ from it. ${ }^{8}$ Anyway, it is assured that the unit-elasticity effect always dominates the ratio effect as long as a normal good is analyzed.

In fact the ratio effect consists of the two effects:

$$
\underbrace{\frac{d \theta}{p_{1}} y}_{\text {ratio effect }}=\underbrace{\left.\frac{d q_{1}}{d p_{1}}\right|_{p_{2}, v=\text { const }}}_{\text {substitution effect }}+\underbrace{\left.\frac{p_{2}}{p_{1}} q_{1} \frac{d q_{2}}{d y}\right|_{p_{1}, p_{2}=\text { const }}}_{\text {transfer effect }},
$$

where

$$
\begin{aligned}
\left.\frac{d q_{1}}{d p_{1}}\right|_{p_{2}, v=\mathrm{const}} & =-\frac{u_{1} u_{2}^{2}}{p_{1}|U|}<0 \text { and }\left.\frac{p_{2}}{p_{1}} q_{1} \frac{d q_{2}}{d y}\right|_{p_{1}, p_{2}=\mathrm{const}} \\
& =\frac{p_{2}}{p_{1}} q_{1} \frac{u_{2}}{p_{2}} \frac{u_{1} u_{12}-u_{2} u_{11}}{|U|}>0,
\end{aligned}
$$

all partial derivatives and $q_{1}$ being evaluated at $\left(q_{1}^{*}, q_{2}^{*}\right)$. The substitution effect of (8) is the same as that of the Slutsky equation (2). The second effect on the right-hand side of (8) is interpreted as follows. A unit increase in $p_{1}$ decreases income $y$ by $q_{1}$ which leads to a decrease in the demand for $\operatorname{good} 2$ by $^{9}$

$$
\left.q_{1} \frac{d q_{2}}{d y}\right|_{p_{1}, p_{2}=\mathrm{const}}\left(=q_{1} \frac{u_{2}}{p_{2}} \frac{u_{1} u_{12}-u_{2} u_{11}}{|U|}>0\right) .
$$

In nominal terms it has the value multiplied by $p_{2}$. That amount in turn is added to the expenditure on good 1 . That is, a rise in $p_{1}$ caused the demand for good 2 to "transfer" to that for good 1. Correctly speaking, the demand for good 1 increases by the "transfer" effect indicated in (8). ${ }^{10}$

The sign of the ratio effect is indeterminate because it is the sum of the two terms with opposite signs. When those two terms almost cancel out, the ratio effect is negligible. In such a situation, the unit-elasticity effect dominates. This is an important message the new Eq. (7) sends.

\footnotetext{
8 "Appendix 2" shows a direct method of calculating the ratio effect (8).

9 Remember that good 2 is assumed to be a normal good.

10 As is seen from (7) and (8), it is necessary that the transfer effect is extraordinarily large in order for good 1 to become a Giffen good. In other words, a Giffen good cannot exist without the sacrifice of other goods. This is related to what Silberberg and Walker (1984) emphasized, though they focused on an additively separable utility function with a "pseudo-Giffen good". Using the new Eq. (7), the case of a Giffen good will be analyzed in Sect. 4 .
} 
In order to show the usefulness of it, let us apply it to the above examples. As for the Cobb-Douglas-type utility function (3), Eq. (7) becomes

$$
\begin{aligned}
\underbrace{-\frac{a}{a+b} \frac{y}{p_{1}^{2}}}_{\text {price effect }} & =\underbrace{0}_{\text {ratio effect }}+\underbrace{\left(-\frac{a}{a+b} \frac{y}{p_{1}^{2}}\right)}_{\text {unit-elasticity effect }} \\
& =\underbrace{-\frac{a b}{(a+b)^{2}} \frac{y}{p_{1}^{2}}}_{\text {substitution effect } \ominus}+\underbrace{\frac{a b}{(a+b)^{2}} \frac{y}{p_{1}^{2}}}_{\text {transfer effect } \oplus}+\underbrace{\left(-\frac{a}{a+b} \frac{y}{p_{1}^{2}}\right)}_{\text {unit-elasticity effect } \ominus}<0 .
\end{aligned}
$$

In this case the substitution effect and the transfer effect just cancel out. As a result, the ratio effect vanishes. The price effect is equal to the unit-elasticity effect. So, the price elasticity of demand for good 1 is one, though this fact is well known.

Equation (7) for (5) is given by

$$
\begin{aligned}
\underbrace{-\frac{1+\sigma c}{(1+c)^{2}} \frac{y}{p_{1}^{2}}}_{\text {price effect }} & =\underbrace{-\frac{(\sigma-1) c}{(1+c)^{2}} \frac{y}{p_{1}^{2}}}_{\text {ratio effect }}+\underbrace{\left(-\frac{1}{1+c} \frac{y}{p_{1}^{2}}\right)}_{\text {unit-elasticity effect }} \\
& =\underbrace{-\frac{\sigma c}{(1+c)^{2}} \frac{y}{p_{1}^{2}}}_{\text {substitution effect } \ominus}+\underbrace{\frac{c}{(1+c)^{2}} \frac{y}{p_{1}^{2}}}_{\text {transfer effect } \oplus}+\underbrace{\left(-\frac{1}{1+c} \frac{y}{p_{1}^{2}}\right)}_{\text {unit-elasticity effect } \ominus}<0 .
\end{aligned}
$$

The CES utility function is characterized by the elasticity of substitution $\sigma$. It is known that for $\sigma>1(<1)$ the expenditure on good 1 decreases (increases) as $p_{1}$ goes up. The above equation confirms it because for $\sigma>1(<1)$ the ratio effect is negative (positive) and therefore the price elasticity of demand is greater than (less than) one. And a new result: $\sigma$ is always the ratio of the substitution effect in absolute value to the transfer effect. If $\sigma$ is greater than unity, the substitution effect exceeds the transfer effect. $^{11}$

Finally, it should be noticed that the sum of the transfer effect and the unit-elasticity effect is equal to the income effect in the Slutsky equation (2). This can be checked

\footnotetext{
11 Examples (3) and (5) were originally derived respectively by Cobb et al. (1928) and Arrow et al. (1961) as aggregate production functions. Despite the claim of the latter that empirically $\sigma$ (as the elasticity of substitution between capital and labor) is significantly less than unity, it is now believed by macroeconomists that there is the law that $\sigma$ is almost one. Solow (2005) calls such a situation the "addiction of theorists to Cobb-Douglas". In this sense the Cobb-Douglas production function is more than a mere special case of the CES. See also Douglas (1967) for the triumph. How about (3) and (5) as utility functions? Judging from very frequent use of (3) or a qualitatively equivalent log utility function, it seems that microeconomists also deem that in many cases it is appropriate to put it equal to unity. It applies to $\sigma$ as the intertemporal elasticity of substitution in consumption, too. But Hall (1988) estimates that it is close to zero.
} 
by comparing general formulations (2) and (7). It is also seen at once by looking at the above Eqs. (9) and (10) derived from the two examples.

\section{Graphical Representations}

In the previous section, a new equation decomposing the price effect was explained in detail by mathematical expressions and concrete examples. They made economists know well how new and how useful it is, I believe. But graphical representations are convenient for anyone. In this section, the new decomposition is explained further using three figures.

Figure 1 shows how such a decomposition of the price effect is drawn on the $q_{1} q_{2}$ plane. When $p_{1}=p_{1}^{\prime}$ and $p_{2}=p_{2}^{\prime}$, this consumer chooses an optimal combination $\left(q_{1}^{*}, q_{2}^{*}\right)$ of goods 1 and 2 . It is indicated by $\bullet$ as the point at which an upper budget line is tangent to a right indifference curve. Next assume that $p_{1}$ rises from $p_{1}^{\prime}$ to $p_{1}^{\prime \prime}$ while $p_{2}=p_{2}^{\prime}$. Then, the budget line of this consumer pivots clockwise on the $q_{2}$-intercept. A new optimal combination $\left(q_{1}^{* *}, q_{2}^{* *}\right)$ is found as the point where the lower budget line touches a left indifference curve.

As shown in the figure, the price effect $(P E)$ is measured by the difference between $q_{1}^{*}$ and $q_{1}^{* *}$. According to (7) the price effect is divided into the ratio effect and the unit-elasticity effect. Such a relation is pictured by the coupling of two arrows. One is the westward arrow starting at the initial optimal point. Note that this arrow runs horizontally. It means that the ordinate is still $q_{2}^{*}$ at the head of it. Furthermore $p_{2}$ does not change, either. Thus, this arrow exactly represents the unit-elasticity effect

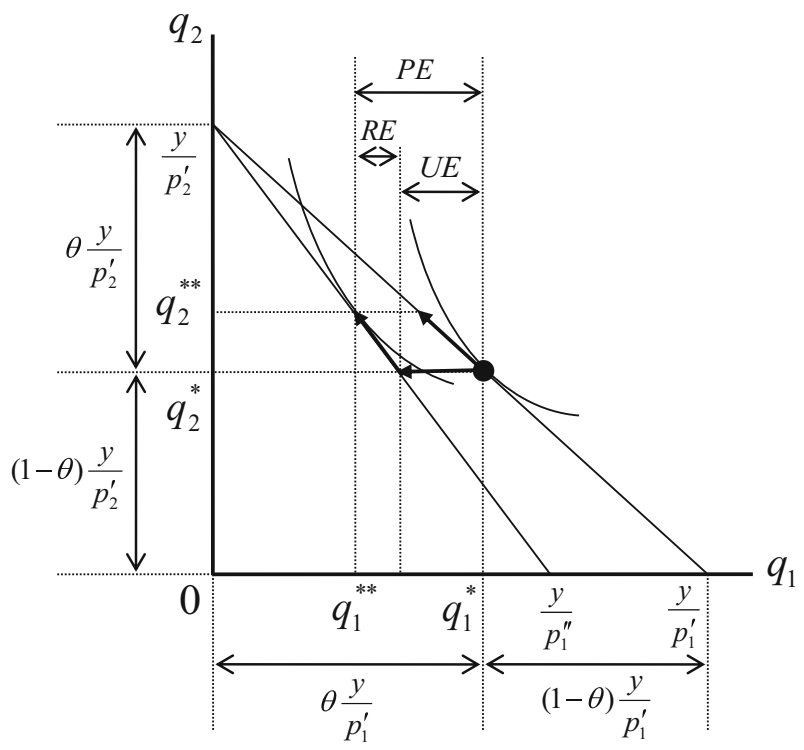

Fig. 1 A decomposition of the price effect: the case of a negative ratio effect. $P E$ price effect, $R E$ ratio effect, $U E$ unit-elasticity effect. $P E=R E+U E$ 


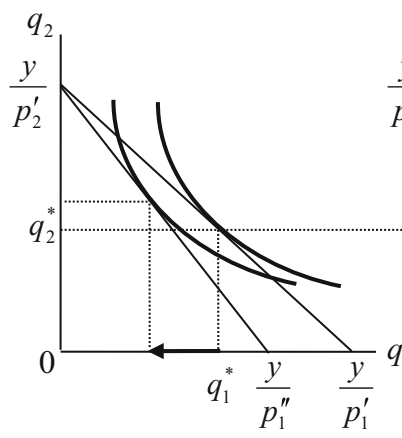

(a)

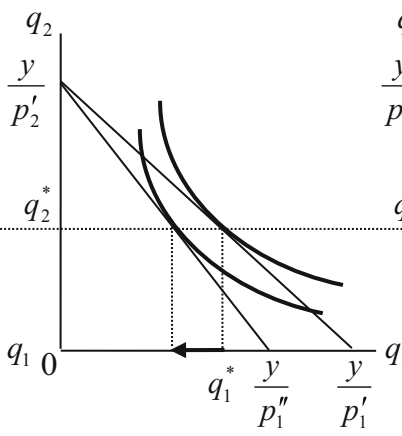

(b)

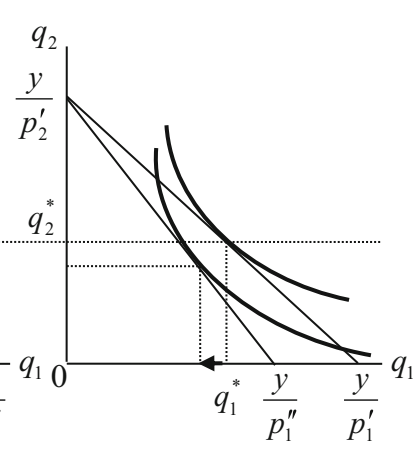

(c)

Fig. 2 Three types of indifference curves. a Negative ratio effect, $\mathbf{b}$ zero ratio effect, $\mathbf{c}$ positive ratio effect

$(U E)$. The other is the arrow running northwestward along the lower budget line and reaching the new optimal point. It approximately shows the ratio effect $(R E){ }^{12}$

In Fig. 1 the ratio effect is negative since the demand for good 1 decreases along the northwestward arrow as $p_{1}$ goes up. The ratio effect may be zero or positive. As is easily seen, when the ratio effect is positive, the arrow starting at the head of the westward arrow runs southeastward along the lower budget line. The zero ratio effect means the disappearance of an arrow running along the budget line. In Fig. 2, depending on the signs of the ratio effect, three types of indifference curves are depicted on the $q_{1} q_{2}$ plane. The Cobb-Douglas-type utility function corresponds exactly to type b. And the CES utility function with $\sigma$ greater than (less than) unity belongs to type a (type c). ${ }^{13}$

So far the demand for good 1 was examined on the $q_{1} q_{2}$ plane taking into account the connection with the demand for good 2. No doubt it is a right way to analyze it. But people eventually want (visually more familiar) demand curves for good 1 on the $q_{1} p_{1}$ plane, do not they? Then, how do the above arguments based on a new decomposition (7) are related to ordinary demand curves? The answer is condensed into Fig. 3, where three types of demand curves are drawn on the $q_{1} p_{1}$ plane, corresponding to three types of indifference curves in Fig. 2.

\footnotetext{
12 Strictly speaking, the ratio effect is represented by the arrow starting at the initial optimal point and running northwestward along the upper budget line. For $p_{1}$ in the denominator of the first term on the right-hand side of (7) takes a value before it rises. In this case it is $p_{1}^{\prime}$, not $p_{1}^{\prime \prime}$. Such an arrow is also depicted in Fig. 1. The two northwestward arrows almost coincide when variations in $p_{1}$ are small enough.

13 Instead of the direction of an arrow, the sign of the ratio effect can also be discerned graphically with the help of "triangles." Look at Fig. 1 again and call points $\left(y / p_{2}^{\prime}, 0\right),(0,0),\left(0, y / p_{1}^{\prime}\right)$, and $\left(q_{1}^{*}, q_{2}^{*}\right)$ respectively points $A, B, C$, and $D$. Then it is easy to understand that the ratio of the area of triangle $A B D$ to that of right triangle $A B C$ is equal to the ratio of income $y$ that goes to the purchase of good 1 for $p_{1}=p_{1}^{\prime}$, say, $\theta$. In addition call points $\left(y / p_{1}^{\prime \prime}, 0\right)$ and $\left(q_{1}^{* *}, q_{2}^{* *}\right)$ respectively points $C^{\prime}$ and $D^{\prime}$. Then the ratio of the area of triangle $A B D^{\prime}$ to that of right triangle $A B C^{\prime}$ is equal to the ratio of income $y$ that goes to the purchase of good 1 for $p_{1}=p_{1}^{\prime \prime}$, say, $\theta^{\prime}$. Note that right-angled triangles $A B C$ and $A B C^{\prime}$ have side $A B$ in common. It follows that $\theta>\theta^{\prime}$, i.e., the ratio effect is negative, if and only if point $D^{\prime}$ is above horizontal line $q_{2}=q_{2}^{*}$ as in Fig. 1 or 2a. Similarly, the ratio effect is positive if and only if point $D^{\prime}$ is below horizontal line $q_{2}=q_{2}^{*}$ as in Fig. 2c. It is only when points $D$ and $D^{\prime}$ are both on the same horizontal line that the ratio effect is zero as in Fig. $2 b$.
} 


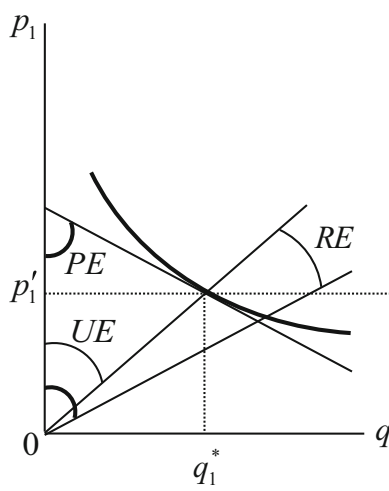

(a)

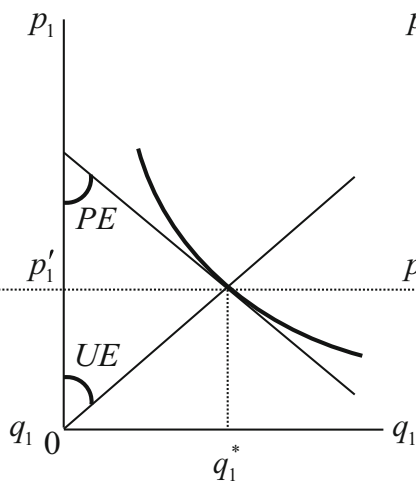

(b)

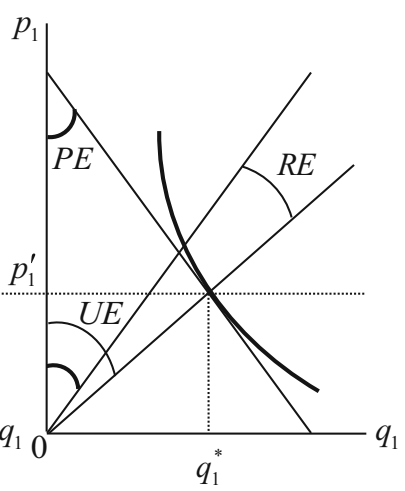

(c)

Fig. 3 Three types of demand curves. See Fig. 1. a Negative ratio effect, b zero ratio effect, c positive ratio effect

For example, look at a demand curve of type a, the flattest one of three types. The demand for good 1 is $q_{1}^{*}$ for $p_{1}=p_{1}^{\prime}$. The price effect at this point is measured by the angle formed by the vertical axis and the tangential line of the demand curve at the same point. It is indicated by a bold arc $P E$. The unit-elasticity effect, the magnitude of which is $q_{1}^{*} / p_{1}^{\prime}$, is represented by the angle formed by the vertical axis and the straight line starting at the origin and passing through point $\left(q_{1}^{*}, p_{1}^{\prime}\right)$. The reason is straightforward. The arc $U E$ shows the unit-elasticity effect. Call the straight line characterized by the arc the $U E$ line. To detect the ratio effect, draw a straight line from the origin such that the vertical axis and the straight line form the angle with the same size as the price effect. Such an angle is indicated by a bold arc near the origin. And call the straight line the $P E$ line. Since the ratio effect is negative in type a, the price effect is greater than the unit-elasticity effect in absolute value by the ratio effect also in absolute value. ${ }^{14}$ Thus, on the $q_{1} p_{1}$ plane the slope of the $P E$ line is less than that of the $U E$ line by the ratio effect, as shown by the arc $R E$.

A demand curve of type $\mathrm{c}$ can be seen in a similar way. The only difference is that the slope of the $P E$ line is greater than that of the $U E$ line by the ratio effect as also shown by the arc $R E$. Finally the ratio effect vanishes and the $P E$ line coincides with the $U E$ line in a demand curve of type b. A demand curve which is always of type $b$ is none other than a rectangular hyperbola.

As has been seen from Fig. 3, a very convenient feature of this new decomposition is that it can be expressed directly on the $q_{1} p_{1}$ plane, using a demand curve in question. ${ }^{15}$ This is not possible for the Slutsky decomposition which can be illustrated only on the $q_{1} q_{2}$ plane. Furthermore, the decompositions in Fig. 3 basically obtain in the n-good

\footnotetext{
14 The relationship among the three effects are the same as that in Fig. 1.

15 Take as a familiar example a linear demand function $p_{1}=-\alpha q_{1}+\beta, \alpha, \beta>0$ which is often used in monopoly theory. The price effect for good 1 is always $-1 / \alpha$ in this case. But the demand curve belongs to all three types, i.e., it is of type a (b, c) for $p_{1}$ greater than (equal to, less than) $\beta / 2$. It can be understood at once by drawing the graph on the $q_{1} p_{1}$ plane.
} 
case, too. The unit-elasticity effect is always found with the help of a straight line connecting the origin and a relevant point like $\left(q_{1}^{*}, p_{1}^{\prime}\right)$. If the ratio effect is zero, the unit-elasticity effect thus found is also the price effect even in the n-good case! The slope of a demand curve differs from that of a rectangular hyperbola according as the ratio effect is negative or positive. That is, the slope becomes flatter (less flat) for a negative (positive) ratio effect. And it is the ratio effect that such a difference means even in the n-good case!

\section{A Giffen Good}

Hicks (1939) (p. 35) stated about the law of demand as follows: "Exceptions to it are rare and unimportant". Stigler $(1947,1987)$ also continued to negate the existence of a Giffen good all his life. Nevertheless, there have been persistent efforts to find Giffen goods empirically and theoretically. Empirically, for example, Battalio et al. (1991) confirmed the existence of a Giffen good for rats, while recently Jensen and Miller (2008) reported the "first real-world evidence of Giffen behavior". Theoretical interests lay in specifying utility functions which gave rise to Giffen behavior. Such examples were submitted, e.g., by Wold (1948), Vandermeulen (1972), Spiegel (1994), Moffatt (2002), and Doi et al. (2009).

From a theoretical point of view, the utility function constructed by Doi et al. (2009) is noteworthy because of its beauty and tractability. Using the notations of this paper, their utility function can be written as

$$
u\left(q_{1}, q_{2}\right)=a \log q_{1}+b \log q_{2}-c q_{1} q_{2}, \quad b>a>b / 2>0, c>0
$$

with $a, b$, and $c$ as positive parameters. Note that it is $c$ that makes the utility function (11) different from the Cobb-Douglas (3). If $c=0$, then (11) is virtually the same as (3). Without resort to the Slutsky equation but by examining the relationship between (11) and a budget constraint in depth, they found out that good 1 becomes a Giffen good for $b-\sqrt{\frac{1}{2} b(b-a)}<c q_{1} q_{2}<a$.

Their stunning result can be interpreted in two ways. First, the Slutsky equation decomposes the price effect into the substitution effect and the income effect as follows:

$$
\underbrace{\left.\frac{d q_{1}}{d p_{1}}\right|_{p_{2}, y=\text { const }}}_{\text {price effect }}=\underbrace{-\frac{u_{1} u_{2}^{2}}{p_{1}|U|}}_{\text {substitution effect } \ominus}+\underbrace{\left[-\frac{u_{1}\left(c^{2} q_{1}^{2} q_{2}^{2}-2 b c q_{1} q_{2}+a b\right)}{p_{1} q_{2}^{2}|U|}\right]}_{\text {income effect } \oplus}>0,
$$

where all partial derivatives, $q_{1}$, and $q_{2}$ are evaluated at the interior optimal point. Simply speaking, the condition $b-\sqrt{\frac{1}{2} b(b-a)}<c q_{1} q_{2}<a$ helps to render the income effect positive enough. The Slutsky equation (12) means that the income effect is so large that it overwhelms the substitution effect with the result that the price effect takes a positive value. Needless to say, this is a conventional explanation for a Giffen good. 
Second, the new equation decomposes the price effect into the ratio effect and the unit-elasticity effect as follows:

$$
\underbrace{\left.\frac{d q_{1}}{d p_{1}}\right|_{p_{2}, y=\text { const }}}_{\text {price effect }}=\underbrace{\frac{(b-a) c u_{2}}{p_{1}|U|}}_{\text {ratio effect } \oplus}+\underbrace{\left(-\frac{q_{1}}{p_{1}}\right)}_{\text {unit-elasticity effect } \ominus}>0 .
$$

Unlike the traditional Slutsky equation, the new one (13) says that a positive price effect is due to the predominance of a positive ratio effect over a negative unit-elasticity effect. Judging from the above decomposition, it is necessary for $c$ to be relatively large in order for the ratio effect to overpower the unit-elasticity effect. And that is actually what Lemma 4.1 in Doi et al. (2009) (p. 256) implies. ${ }^{16}$

\section{Cross-Price Effect}

It is interesting to examine the relation between the new decomposition and the Slutsky decomposition of the cross-price effect. Such a version of the Slutsky equation says the cross-price effect with respect to good 1 is expressed as the sum of the crosssubstitution effect and the income effect as follows:

$$
\underbrace{\left.\frac{d q_{1}}{d p_{2}}\right|_{p_{1}, y=\text { const }}}_{\text {cross-price effect }}=\underbrace{\left.\frac{d q_{1}}{d p_{2}}\right|_{p_{1}, v=\text { const }}}_{\text {cross-substitution effect }}+\underbrace{\left(-\left.q_{2} \frac{d q_{1}}{d y}\right|_{p_{1}, p_{2}=\text { const }}\right)}_{\text {income effect }},
$$

16 A high value must be put on the efforts made by Doi et al. (2009) for the analysis of the utility function (11). Nevertheless, I think that the specification of utility functions with a Giffen good is still a hard work. The reason is that economists must follow a utility function (1) which is a function of the quantities of goods 1 and 2 only. If the rule is loosened, however, an upward sloping demand curve is easy to draw. Think of a kind of the quasilinear utility function $u=\log q_{1}+\left(y / p_{1}\right)^{2} q_{2}, y>\sqrt[3]{p_{1}^{2} p_{2}}$. Then, the demand for good 1 turns out to be an increasing function of its own price as $q_{1}=p_{1} p_{2} / y^{2}$. Note also that it is an inferior good as well. In this sense it is not a good for conspicuous consumption, and so the demand for it does not come from the Veblen effect which also gives birth to an upward sloping demand curve, as suggested by Leibenstein (1950).

The above utility function and the resulting demand function correspond well to economists' explanation for why Giffen goods exist. For example, Whitehead (2010) (p. 49) says, "This classification of commodities as inferior or Giffen typically fits the basic staples in foodstuffs consumed by the poorer sections of the society. As real income rises and consumers become more affluent, they tend to shift away from the more traditional food staples and towards commodities that were previously priced out of their reach". $y^{2} /\left(p_{1} p_{2}\right)$ in my example can be regarded as (a square of) "real income" the increase of which causes consumer's shift from an inferior good $q_{1}$ to a superior one $q_{2}$. A typical consumer Jensen and Miller (2008) investigated theoretically has also a utility function which apparently depends on her income level (see particularly the subsistence zone in Fig. 1 therein). Doi et al. (2009) showed that Giffen behavior can arise for a relatively low value of $p / I^{2}$. In fact their $I^{2} / p$, the reciprocal of $p / I^{2}$, is just the same as my $y^{2} /\left(p_{1} p_{2}\right)$ because $I$ and $p$ correspond respectively to $y / p_{2}$ and $p_{1} / p_{2}$. The role of income level is important in their arguments for a Giffen good, too. 
where

$$
\begin{aligned}
\left.\frac{d q_{1}}{d p_{2}}\right|_{p_{1}, v=\mathrm{const}} & =\frac{u_{1} u_{2}^{2}}{p_{2}|U|}>0 \text { and }-\left.q_{2} \frac{d q_{1}}{d y}\right|_{p_{1}, p_{2}=\mathrm{const}} \\
& =-q_{2} \frac{u_{2}\left(u_{2} u_{12}-u_{1} u_{22}\right)}{p_{2}|U|}<0,
\end{aligned}
$$

all partial derivatives and $q_{2}$ being evaluated at $\left(q_{1}^{*}, q_{2}^{*}\right)$. Like (2) this equation is also derived by solving Problem I. The cross-substitution is always positive, while the income effect is always negative. Thus the sign of the cross-price effect is indeterminate. But this is a "normal" situation.

Next consider Problem II again. This time differentiate $q_{1}=\frac{\theta y}{p_{1}}$ partially with respect to $p_{2}$. Then a new equation for the cross-price effect is obtained as follows:

$$
\underbrace{\left.\frac{d q_{1}}{d p_{2}}\right|_{p_{1}, y=\text { const }}}_{\text {cross-price effect }}=\underbrace{\frac{\frac{d \theta}{d p_{2}} y}{p_{1}}}_{\text {ratio effect }},
$$

where

$$
\frac{\frac{d \theta}{d p_{2}} y}{p_{1}}=\frac{u_{2}}{p_{2}} \frac{u_{1} u_{2}}{|U|}-q_{2} \frac{u_{2}}{p_{2}} \frac{u_{2} u_{12}-u_{1} u_{22}}{|U|} \gtreqless 0,
$$

all partial derivatives and $q_{2}$ being evaluated at $\left(q_{1}^{*}, q_{2}^{*}\right)$. It is found from (15) that there is no unit-elasticity effect in the cross-price case. Thus, the cross-price effect is governed completely by the ratio effect. But the ratio effect consists of the positive cross-substitution effect and the negative income effect just as the Slutsky equation (14) shows. ${ }^{17}$ Therefore, the sign of the ratio effect is indeterminate, too.

Let us apply Eq. (15) to the Cobb-Douglas-type utility function (3). Then,

$$
\begin{aligned}
\underbrace{0}_{\text {cross-price effect }} & =\underbrace{0}_{\text {ratio effect }} \\
& =\underbrace{\frac{a b}{(a+b)^{2}} \frac{y}{p_{1} p_{2}}}_{\text {cross-substitution effect } \oplus}+\underbrace{\left[-\frac{a b}{(a+b)^{2}} \frac{y}{p_{1} p_{2}}\right]}_{\text {income effect } \ominus}=0 .
\end{aligned}
$$

In this case the cross-substitution effect and the income effect cancel out exactly. As a result, the ratio effect and the cross-price effect are zero. The demand for good 1 is unaffected by variations in price $p_{2}$ of good 2 .

17 "Appendix 3" shows a direct method of calculating the ratio effect in the cross-price case. 


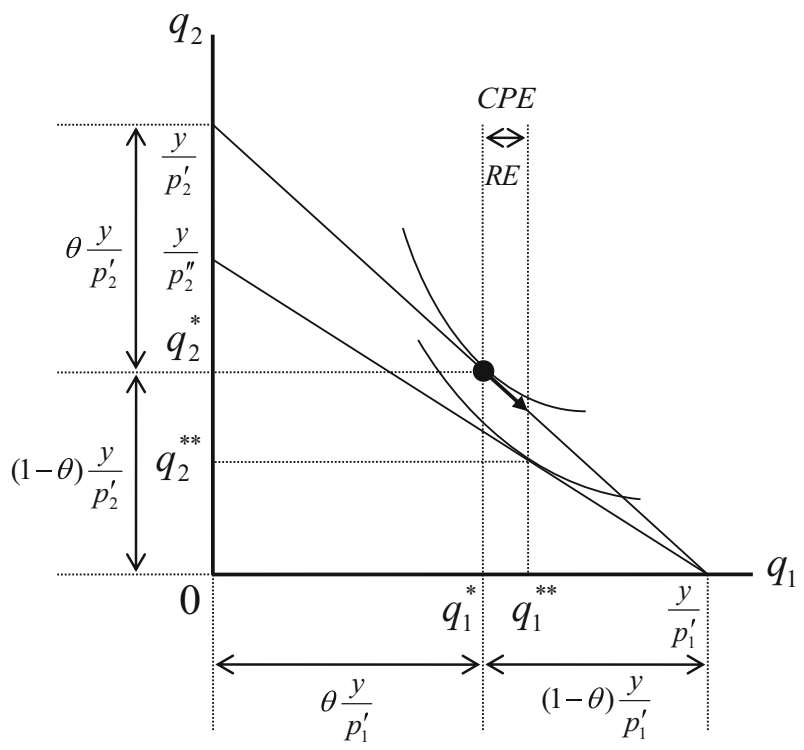

Fig. 4 The cross-price effect. $C P E$ cross-price effect, $R E$ ratio effect. $C P E=R E$

As for the CES utility function (5), Eq. (15) is written as follows:

$$
\begin{aligned}
\underbrace{\frac{(\sigma-1) c}{(1+c)^{2}} \frac{y}{p_{1} p_{2}}}_{\text {cross-price effect }} & =\underbrace{\frac{(\sigma-1) c}{(1+c)^{2}} \frac{y}{p_{1} p_{2}}}_{\text {ratio effect }} \\
& =\underbrace{\frac{\sigma c}{(1+c)^{2}} \frac{y}{p_{1} p_{2}}}_{\text {cross-substitution effect } \oplus}+\underbrace{\left[-\frac{c}{(1+c)^{2}} \frac{y}{p_{1} p_{2}}\right]}_{\text {income effect } \ominus} \gtreqless 0 .
\end{aligned}
$$

It follows that for $\sigma>1(<1)$ the expenditure on good 1 increases (decreases) as $p_{2}$ rises. It is the ratio effect. And, since $p_{1}$ and $y$ remain constant, the demand for good 1 increases (decreases). It is the cross-price effect. ${ }^{18}$

In Fig. 4 is illustrated the cross-price effect for good 1 when $p_{2}$ rises from $p_{2}^{\prime}$ to $p_{2}^{\prime \prime}$ while $p_{1}=p_{1}^{\prime}$. The ratio effect is represented by an arrow starting at the initial optimal

18 The saving decision problem can be solved by the application of Eq. (15). Suppose that $q_{1}$ and $q_{2}$ are current consumption and future consumption, respectively. Moreover, put $p_{1}=1$ and $p_{2}=1 /(1+r)$ with $r$ as the real interest rate. Then, saving $s$ is defined as $y-q_{1}$, and so $1-\theta$ can be regarded as the saving rate. And the response of saving to variations in the interest rate can be written as

$$
\frac{\partial s}{\partial r}=\frac{1}{(1+r)^{2}} \frac{d \theta}{d p_{2}} y .
$$

That is, analysis of saving is tantamount to that of the ratio effect. Empirically it is a well-known (but frequently ignored) fact that the influence of the interest rate on saving is unimportant. See, e.g., Stiglitz and Walsh (2006) (p. 537). 
Price Effect $\left\{\begin{array}{l}\text { Ratio Effect } \\ \text { Unit-elasticity Effect }\end{array}\right\}$ Transfer Effect $\}$ Income Effect

Cross-Price Effect $=$ Ratio Effect $\left\{\begin{array}{l}\text { Cross-Substitution Effect } \\ \text { Income Effect }\end{array}\right.$

Fig. 5 The relation with the Slutsky decomposition

point $\left(q_{1}^{*}, q_{2}^{*}\right)$ and running southwestward along the budget line. Since the demand for good 1 increases, the cross-price effect is positive as in the case of the CES utility function with $\sigma$ greater than unity. Of course, such an arrow points northwestward when the cross-price effect is negative, and it disappears when the cross-price effect vanishes. $^{19}$

Finally it is convenient to summarize the results obtained so far. Figure 5 shows the relation between the new decomposition and the traditional Slutsky decomposition for the price effect and the cross-price effect. First, the price effect can be divided into the ratio effect and the unit-elasticity effect. The latter effect is always negative, whereas the sign of the former effect is indeterminate because it consists of the two effects with opposite signs, the negative (own-)substitution effect and the positive transfer effect. The sum of the transfer effect and the unit-elasticity effect leads to the negative income effect of the Slutsky decomposition. Second, there is no unit-elasticity effect in the cross-price case. The cross-price effect is equal to the ratio effect which in this case is the sum of the two effects of the Slutsky decomposition, i.e., the positive cross-substitution effect and the negative income effect. The sign of the ratio effect is indeterminate in this case, too.

\section{A Case Where a Consumer Holds Initial Endowments}

In this section a case in which a consumer holds initial endowments as in a pure exchange model is examined. Now the income of a consumer is not fixed but varies with prices $p_{1}$ and $p_{2}$.

\footnotetext{
19 In fact, you have already seen these three patterns of the cross-price effect in Fig. 2 as the relationship between price $p_{1}$ of good 1 and the demand for good 2 .
} 


\subsection{Price Effect}

Problem I in Sect. 2 must be rewritten as

$$
\begin{aligned}
& \text { Problem I' }: \max _{q_{1}, q_{2}} u\left(q_{1}, q_{2}\right) \\
& \text { s.t. } p_{1} q_{1}+p_{2} q_{2}=y, \\
& y=p_{1} \bar{q}_{1}+p_{2} \bar{q}_{2},
\end{aligned}
$$

where $\bar{q}_{1}$ and $\bar{q}_{2}$ are respectively non-negative initial endowments of goods 1 and 2 . Solving it yields the Slutsky equation with initial endowments:

$$
\underbrace{\left.\frac{d q_{1}}{d p_{1}}\right|_{p_{2}=\text { const }}}_{\text {price effect }}=\underbrace{\left.\frac{d q_{1}}{d p_{1}}\right|_{p_{2}, v=\text { const }}}_{\text {substitution effect }}+\underbrace{\left[\left.\left(\bar{q}_{1}-q_{1}\right) \frac{d q_{1}}{d y}\right|_{p_{1}, p_{2}=\text { const }}\right]}_{\text {income effect }},
$$

where

$$
\begin{aligned}
\left.\frac{d q_{1}}{d p_{1}}\right|_{p_{2}, v=\mathrm{const}} & =-\frac{u_{1}}{p_{1}} \frac{u_{2}^{2}}{|U|}<0 \text { and }\left.\left(\bar{q}_{1}-q_{1}\right) \frac{d q_{1}}{d y}\right|_{p_{1}, p_{2}=\mathrm{const}} \\
& =\left(\bar{q}_{1}-q_{1}\right) \frac{u_{1}}{p_{1}} \frac{u_{2} u_{12}-u_{1} u_{22}}{|U|} \gtreqless 0,
\end{aligned}
$$

all partial derivatives and $q_{1}$ being evaluated at the optimal point $\left(q_{1}^{*}, q_{2}^{*}\right)$. Note that the assumption that good 1 is a normal good does not warrant the negativity of the price effect any more. It may be zero or positive for $q_{1}^{*}<\bar{q}_{1}$.

Next rewrite Problem II so as to deal with initial endowments $\left(\bar{q}_{1}, \bar{q}_{2}\right)$ as

$$
\begin{aligned}
& \text { Problem II' } \max _{\theta} u\left(q_{1}, q_{2}\right) \\
& \qquad \begin{array}{l}
\text { s.t. } q_{1}=\frac{\theta y}{p_{1}}, q_{2}=\frac{(1-\theta) y}{p_{2}}, \quad 0 \leq \theta \leq 1, \\
y=p_{1} \bar{q}_{1}+p_{2} \bar{q}_{2} .
\end{array}
\end{aligned}
$$

Differentiating $q_{1}=\frac{\theta y}{p_{1}}$ partially with respect to $p_{1}$, taking account of $y=p_{1} \bar{q}_{1}+$ $p_{2} \bar{q}_{2}$, gives the following equation:

$$
\underbrace{\left.\frac{d q_{1}}{d p_{1}}\right|_{p_{2}=\text { const }}}_{\text {price effect }}=\underbrace{\frac{d \theta}{d p_{1}} y}_{\text {ratio effect }}+\underbrace{p_{1}}_{\text {constant-ratio effect }}+\underbrace{\left(\left.\frac{d q_{1}}{d p_{1}}\right|_{\theta=}\right)}_{\theta=\text { const }}
$$


where

$$
\begin{aligned}
\frac{\frac{d \theta}{d p_{1}} y}{p_{1}}= & -\frac{u_{1}}{p_{1}} \frac{u_{2}^{2}}{|U|}+\frac{q_{1}}{p_{1}} \frac{u_{1} u_{2} u_{12}-u_{2}^{2} u_{11}}{|U|}+\frac{\bar{q}_{1} q_{1}}{y} \frac{u_{2}^{2} u_{11}-u_{1} u_{2} u_{12}}{|U|} \\
& +\frac{\bar{q}_{1} q_{2}}{y} \frac{u_{2}^{2} u_{12}-u_{1} u_{2} u_{22}}{|U|} \gtreqless 0
\end{aligned}
$$

and

$$
\left.\frac{d q_{1}}{d p_{1}}\right|_{\theta=\mathrm{const}}=-\frac{p_{2} \bar{q}_{2}}{y} \frac{q_{1}}{p_{1}} \leq 0,
$$

$q_{1}, q_{2}$, and all partial derivatives being evaluated at $\left(q_{1}^{*}, q_{2}^{*}\right)$.

According to (19), the price effect with initial endowments can be divided into the "ratio" effect and the "constant-ratio" effect. Equation (19) is not the same as Eq. (7) with $y$ as given, though they resembles each other. The "constant-ratio effect" in (19) represents how the demand for good 1 changes in response to a rise in its own price if the ratio $\theta$ of expenditure for the good to income remains constant. The magnitude of it can be calculated from the relation $p_{1} q_{1}=\theta\left(p_{1} \bar{q}_{1}+p_{2} \bar{q}_{2}\right)$ with $\theta$ as constant. Total differentiation of it gives the value of $-\frac{p_{2} \bar{q}_{2}}{y} \frac{q_{1}}{p_{1}}$. The price elasticity of demand in this situation is $\frac{p_{2} \bar{q}_{2}}{y}$ which lies between zero and one.

The price effect differs from the constant-ratio effect by the ratio effect. The ratio effect indicates how the demand for good 1 changes in response to a rise in its own price if income is kept unchanged. It can be obtained by subtracting $-\frac{p_{2} \bar{q}_{2}}{y} \frac{q_{1}}{p_{1}}$ from the right-hand side of (18). ${ }^{20}$

Remember that the ratio effect in (7) consists of two effects. But the ratio effect in (19) consists of four effects:

$$
\begin{aligned}
& \underbrace{\frac{d \theta}{d p_{1}} y}_{\text {ratio effect }}=\underbrace{\left.\frac{d q_{1}}{d p_{1}}\right|_{p_{2}, v=\text { const }}}_{\text {substitution effect }}+\underbrace{\frac{p_{2}}{p_{1}} q_{1}\left(\left.\frac{d q_{2}}{d y}\right|_{p_{1}, p_{2}=\text { const }}\right)}_{\text {transfer effect }} \\
& \underbrace{-\frac{p_{2}}{p_{1}}\left(\theta \frac{\partial y}{\partial p_{1}}\right)\left(\left.\frac{d q_{2}}{d y}\right|_{p_{1}, p_{2}=\text { const }}\right)}_{\text {first endowment effect }} \\
& +\underbrace{\left[(1-\theta) \frac{\partial y}{\partial p_{1}}\right]\left(\left.\frac{d q_{1}}{d y}\right|_{p_{1}, p_{2}=\mathrm{const}}\right)}, \\
& \text { second endowment effect }
\end{aligned}
$$

\footnotetext{
20 It can also be derived in the same way as in "Appendix 2 " except for the terms relating to $\bar{q}_{1}$.
} 
where

$$
\begin{aligned}
\left.\frac{d q_{1}}{d p_{1}}\right|_{p_{2}, v=\mathrm{const}} & =-\frac{u_{1}}{p_{1}} \frac{u_{2}^{2}}{|U|}<0, \\
\frac{p_{2}}{p_{1}} q_{1}\left(\left.\frac{d q_{2}}{d y}\right|_{p_{1}, p_{2}=\mathrm{const}}\right) & =\frac{p_{2}}{p_{1}} q_{1} \frac{u_{2}}{p_{2}} \frac{u_{1} u_{12}-u_{2} u_{11}}{|U|}>0, \\
-\frac{p_{2}}{p_{1}}\left(\theta \frac{\partial y}{\partial p_{1}}\right)\left(\left.\frac{d q_{2}}{d y}\right|_{p_{1}, p_{2}=\mathrm{const}}\right) & =-\frac{p_{2}}{p_{1}}\left(\frac{p_{1} q_{1}}{y} \bar{q}_{1}\right) \frac{u_{2}}{p_{2}} \frac{u_{1} u_{12}-u_{2} u_{11}}{|U|} \leq 0, \text { and } \\
{\left[(1-\theta) \frac{\partial y}{\partial p_{1}}\right]\left(\left.\frac{d q_{1}}{d y}\right|_{p_{1}, p_{2}=\mathrm{const}}\right) } & =\left(\frac{p_{2} q_{2}}{y} \bar{q}_{1}\right) \frac{u_{1}}{p_{1}} \frac{u_{2} u_{12}-u_{1} u_{22}}{|U|} \geq 0,
\end{aligned}
$$

$q_{1}, q_{2}$, and all partial derivatives being evaluated at $\left(q_{1}^{*}, q_{2}^{*}\right)$. The first two terms on the right-hand side of (20) are virtually the same as those in (8). The remaining two terms, which are called the "first endowment effect" and the "second endowment effect", are proper to a case with initial endowments.

The way to interpret the latter two effects is similar to the explanation of the transfer effect in (8). The first endowment effect represented by the third term is interpreted as follows. A unit increase in $p_{1}$ actually increases the income of a consumer by $\bar{q}_{1}$. The ratio $\theta$ of the increase in income leads to an increase in the demand for good 1 which is already reflected in the constant-ratio effect in (19). On the other hand, the ratio $\theta$ of the increase in income also increases the demand for good 2 by

$$
\left(\theta \frac{\partial y}{\partial p_{1}}\right)\left(\left.\frac{d q_{2}}{d y}\right|_{p_{1}, p_{2}=\mathrm{const}}\right)\left[=\left(\frac{p_{1} q_{1}}{y} \bar{q}_{1}\right) \frac{u_{2}}{p_{2}} \frac{u_{1} u_{12}-u_{2} u_{11}}{|U|} \geq 0\right]
$$

In nominal terms it has the value multiplied by $p_{2}$. That amount is covered by part of the expenditure on good 1 . That is, a rise in $p_{1}$ has the demand for good 1 "transfer" to that for good 2. As a result, the demand for good 1 decreases by the first endowment effect. The ratio $1-\theta$ of the increase in income coming from a unit increase in $p_{1}$ leads to an increase in the demand for good 1 by the second endowment effect.

The constant-ratio effect is non-positive, but the sign of the ratio effect is indeterminate. Thus, the price effect is ambiguous. Then, the applications of (19) to examples (3) and (5) will help. Equation (19) for the Cobb-Douglas-type utility function (3) becomes

$$
\begin{aligned}
\underbrace{-\frac{a}{a+b} \frac{p_{2} \bar{q}_{2}}{p_{1}^{2}}}_{\text {price effect }} & =\underbrace{0}_{\text {ratio effect }}+\underbrace{\left(-\frac{a}{a+b} \frac{p_{2} \bar{q}_{2}}{p_{1}^{2}}\right)}_{\text {constant-ratio effect }} \\
& =\underbrace{-\frac{a b}{(a+b)^{2}} \frac{y}{p_{1}^{2}}}_{\text {substitution effect } \ominus}+\underbrace{\frac{a b}{(a+b)^{2}} \frac{y}{p_{1}^{2}}}_{\text {transfer effect } \oplus} \underbrace{-\frac{a b}{(a+b)^{2}} \frac{\bar{q}_{1}}{p_{1}}}_{\text {first endowment effect }}
\end{aligned}
$$




$$
\begin{aligned}
& +\underbrace{\frac{a b}{(a+b)^{2}} \frac{\bar{q}_{1}}{p_{1}}}_{\text {second endowment effect }} \underbrace{-\frac{a}{a+b} \frac{p_{2} \bar{q}_{2}}{p_{1}^{2}} \leq 0,}_{\text {constant-ratio effect }} \\
& =\underbrace{-\frac{a b}{(a+b)^{2}} \frac{p_{1} \bar{q}_{1}+p_{2} \bar{q}_{2}}{p_{1}^{2}}}_{\text {substitution effect } \ominus}+\underbrace{\frac{a}{(a+b)^{2}} \frac{b p_{1} \bar{q}_{1}-a p_{2} \bar{q}_{2}}{p_{1}^{2}}}_{\text {income effect }}]
\end{aligned}
$$

where $y=p_{1} \bar{q}_{1}+p_{2} \bar{q}_{2}$ and the last line in big brackets is due to the Slutsky equation (18). The substitution effect and the transfer effect just cancel out as in (9). And, surprisingly, so do the first and the second endowment effects. Hence zero ratio effect. The price effect is governed entirely by the non-positive constant-ratio effect.

The price effect in the case of the CES utility function (5) is decomposed by (19) as follows:

$$
\begin{aligned}
& \underbrace{-\frac{(\sigma-1) c p_{1} \bar{q}_{1}+(\sigma c+1) p_{2} \bar{q}_{2}}{(1+c)^{2}} \frac{1}{p_{1}^{2}}}_{\text {price effect }}=\underbrace{-\frac{(\sigma-1) c}{(1+c)^{2}} \frac{y}{p_{1}^{2}}}_{\text {ratio effect }}+\underbrace{\left(-\frac{1}{1+c} \frac{p_{2} \bar{q}_{2}}{p_{1}^{2}}\right)}_{\text {constant-ratio effect }} \\
& =\underbrace{-\frac{\sigma c}{(1+c)^{2}} \frac{y}{p_{1}^{2}}}_{\text {substitution effect } \ominus}+\underbrace{\frac{c}{(1+c)^{2}} \frac{y}{p_{1}^{2}}}_{\text {transfer effect } \oplus} \underbrace{-\frac{c}{(1+c)^{2} \frac{\bar{q}_{1}}{p_{1}}}}_{\text {first endowment effect }}
\end{aligned}
$$

$$
+\underbrace{\frac{c}{(1+c)^{2}} \frac{\bar{q}_{1}}{p_{1}}}_{\text {second endowment effect }} \underbrace{-\frac{1}{1+c} \frac{p_{2} \bar{q}_{2}}{p_{1}^{2}}}_{\text {constant-ratio effect }} \gtreqless 0,
$$

$$
[\underbrace{-\frac{\sigma c}{(1+c)^{2}} \frac{p_{1} \bar{q}_{1}+p_{2} \bar{q}_{2}}{p_{1}^{2}}}_{\text {substitution effect } \ominus}+\underbrace{\frac{1}{(1+c)^{2}} \frac{c p_{1} \bar{q}_{1}-p_{2} \bar{q}_{2}}{p_{1}^{2}}}_{\text {income effect }}]
$$

where $y=p_{1} \bar{q}_{1}+p_{2} \bar{q}_{2}$ and the last line in big brackets is the Slutsky decomposition (18). Surprisingly again, the first and the second endowment effects cancel out exactly. Thus the ratio effect takes the same form as that in (10). The sign of the price effect is indeterminate, but it can be said that it is non-positive if $\sigma \geq 1$. $^{21}$

\footnotetext{
21 The labor supply decision problem of a worker can be solved by the application of (19). Put $p_{2}=1$ and $\bar{q}_{2}=0$, and think of $q_{1}, \bar{q}_{1}, q_{2}$, and $p_{1}$ respectively as leisure, total available time, consumption, and the (real) wage rate. Then, labor supply $n$ is the difference between $\bar{q}_{1}$ and $q_{1}$, i.e., $n=(1-\theta) \bar{q}_{1} .1-\theta$ can be regarded as the ratio of total available time used for labor. The response of the labor supply to variations in the
} 


\subsection{Cross-Price Effect}

Solving Problem I' also gives the Slutsky equation for the cross-price effect with initial endowments $\left(\bar{q}_{1}, \bar{q}_{2}\right)$ :

$$
\underbrace{\left.\frac{d q_{1}}{d p_{2}}\right|_{p_{1}=\text { const }}}_{\text {cross-price effect }}=\underbrace{\left.\frac{d q_{1}}{d p_{2}}\right|_{p_{1}, v=\text { const }}}_{\text {cross-substitution effect }}+\underbrace{\left[\left.\left(\bar{q}_{2}-q_{2}\right) \frac{d q_{1}}{d y}\right|_{p_{1}, p_{2}=\text { const }}\right]}_{\text {income effect }},
$$

where

$$
\begin{aligned}
\left.\frac{d q_{1}}{d p_{2}}\right|_{p_{1}, v=\mathrm{const}} & =\frac{u_{1} u_{2}^{2}}{p_{2}|U|}>0 \text { and }\left.\left(\bar{q}_{2}-q_{2}\right) \frac{d q_{1}}{d y}\right|_{p_{1}, p_{2}=\mathrm{const}} \\
& =\left(\bar{q}_{2}-q_{2}\right) \frac{u_{2}}{p_{2}} \frac{u_{2} u_{12}-u_{1} u_{22}}{|U|} \gtreqless 0,
\end{aligned}
$$

all partial derivatives and $q_{2}$ being evaluated at the optimal point $\left(q_{1}^{*}, q_{2}^{*}\right)$.

On the other hand a new equation for the cross-price effect with initial endowments can be derived from Problem II'. Differentiating $q_{1}=\frac{\theta y}{p_{1}}$ partially with respect to $p_{2}$, taking account of $y=p_{1} \bar{q}_{1}+p_{2} \bar{q}_{2}$, yields the following equation:

$$
\underbrace{\left.\frac{d q_{1}}{d p_{2}}\right|_{p_{1}=\text { const }}}_{\text {cross-price effect }}=\underbrace{\frac{d \theta}{d p_{2}} y}_{\text {ratio effect }}+\underbrace{\left(\left.\frac{d q_{1}}{d p_{2}}\right|_{\theta=\mathrm{const}}\right)}_{\text {constant-ratio effect }}
$$

where

$$
\begin{aligned}
\frac{\frac{d \theta}{d p_{2}} y}{p_{1}}= & \frac{u_{2}}{p_{2}} \frac{u_{1} u_{2}}{|U|}-q_{2} \frac{u_{2}}{p_{2}} \frac{u_{2} u_{12}-u_{1} u_{22}}{|U|}+\frac{\bar{q}_{2} q_{1}}{y} \frac{u_{2}^{2} u_{11}-u_{1} u_{2} u_{12}}{|U|} \\
& +\frac{\bar{q}_{2} q_{2}}{y} \frac{u_{2}^{2} u_{12}-u_{1} u_{2} u_{22}}{|U|} \gtreqless 0
\end{aligned}
$$

and

$$
\left.\frac{d q_{1}}{d p_{2}}\right|_{\theta=\mathrm{const}}=\frac{p_{2} \bar{q}_{2}}{y} \frac{q_{1}}{p_{2}} \geq 0,
$$

$q_{1}, q_{2}$, and all partial derivatives being evaluated at $\left(q_{1}^{*}, q_{2}^{*}\right)$.

Footnote 21 continued

wage rate can be written as

$$
\frac{\partial n}{\partial p_{1}}=-\frac{d \theta}{d p_{1}} \bar{q}_{1}
$$

Since there is no constant-ratio effect in this problem, labor supply is governed completely by the ratio effect. But I have not heard that it is affected by the wage rate significantly. 
It should be noticed that there is the constant-ratio effect in (22) unlike (15) in which only the ratio effect exists. Even if the ratio effect is zero, the demand for good 1 can respond positively to a change in price $p_{2}$ of good 2 . The constant-ratio effect can be calculated from the relation $p_{1} q_{1}=\theta\left(p_{1} \bar{q}_{1}+p_{2} \bar{q}_{2}\right)$. Differentiating it totally and putting $d p_{1}=d \theta=0$ gives the value of $\frac{p_{2} \bar{q}_{2}}{y} \frac{q_{1}}{p_{2}}$. The "cross-elasticity" of demand in this situation is $\frac{p_{2} \bar{q}_{2}}{y}$ which is between zero and one.

The cross-price effect differs from the constant-ratio effect by the ratio effect. The ratio effect can be obtained by subtracting $\frac{p_{2} \bar{q}_{2}}{y} \frac{q_{1}}{p_{1}}$ from the right-hand side of (21). ${ }^{22}$ The ratio effect is the sum of of the four effects:

$$
\begin{aligned}
\underbrace{\frac{d \theta}{p_{1}} y}_{\text {ratio effect }}= & \underbrace{\left.\frac{d q_{1}}{d p_{2}}\right|_{p_{1}, v=\text { const }}-q_{2}\left(\left.\frac{d q_{1}}{d y}\right|_{p_{1}, p_{2}=\text { const }}\right)}_{\text {cross-substitution effect }} \\
& \underbrace{-\frac{p_{2}}{p_{1}}\left(\theta \frac{\partial y}{\partial p_{2}}\right)\left(\left.\frac{d q_{2}}{d y}\right|_{p_{1}, p_{2}=\text { const }}\right)}_{\text {income effect }} \\
& +\underbrace{\left[(1-\theta) \frac{\partial y}{\partial p_{2}}\right]\left(\left.\frac{d q_{1}}{d y}\right|_{p_{1}, p_{2}=\text { const }}\right)}_{\text {first endowment effect }},
\end{aligned}
$$

where

$$
\begin{aligned}
\left.\frac{d q_{1}}{d p_{2}}\right|_{p_{1}, v=\mathrm{const}} & =\frac{u_{2}}{p_{2}} \frac{u_{1} u_{2}}{|U|}>0, \\
-q_{2}\left(\left.\frac{d q_{1}}{d y}\right|_{p_{1}, p_{2}=\mathrm{const}}\right) & =-q_{2} \frac{u_{2}}{p_{2}} \frac{u_{2} u_{12}-u_{1} u_{22}}{|U|}<0, \\
-\frac{p_{2}}{p_{1}}\left(\theta \frac{\partial y}{\partial p_{2}}\right)\left(\left.\frac{d q_{2}}{d y}\right|_{p_{1}, p_{2}=\mathrm{const}}\right) & =-\frac{p_{2}}{p_{1}}\left(\frac{p_{1} q_{1}}{y} \bar{q}_{2}\right) \frac{u_{2}}{p_{2}} \frac{u_{1} u_{12}-u_{2} u_{11}}{|U|} \leq 0, \text { and } \\
{\left[(1-\theta) \frac{\partial y}{\partial p_{2}}\right]\left(\left.\frac{d q_{1}}{d y}\right|_{p_{1}, p_{2}=\mathrm{const}}\right) } & =\left(\frac{p_{2} q_{2}}{y} \bar{q}_{2}\right) \frac{u_{2}}{p_{2}} \frac{u_{2} u_{12}-u_{1} u_{22}}{|U|} \geq 0,
\end{aligned}
$$

$q_{1}, q_{2}$, and all partial derivatives being evaluated at $\left(q_{1}^{*}, q_{2}^{*}\right)$. The substitution effect and the income effect on the right-hand side of (23) are virtually the same as those in (15). The first and second endowment effects can be interpreted similarly to those in (20).

To show the usefulness of (22), let us apply it to examples (3) and (5). Equation (22) for the Cobb-Douglas-type utility function (3) becomes

$\overline{22}$ Or it can directly be calculated in the same way as in "Appendix 3 " except for the terms relating to $\bar{q}_{2}$. 


$$
\begin{aligned}
& \underbrace{\frac{a}{a+b} \frac{\bar{q}_{2}}{p_{1}}}_{\text {cross-price effect }}=\underbrace{0}_{\text {ratio effect }}+\underbrace{\frac{a}{a+b} \frac{\bar{q}_{2}}{p_{1}}}_{\text {constant-ratio effect }} \\
& =\underbrace{\frac{a b}{(a+b)^{2}} \frac{y}{p_{1} p_{2}}}_{\text {cross-substitution effect } \oplus}-\underbrace{-\frac{a b}{(a+b)^{2}} \frac{y}{p_{1} p_{2}}}_{\text {income effect } \ominus} \underbrace{-\frac{a b}{(a+b)^{2}} \frac{\bar{q}_{2}}{p_{1}}}_{\text {first endowment effect }} \\
& +\underbrace{\frac{a b}{(a+b)^{2}} \frac{\bar{q}_{2}}{p_{1}}}_{\text {second endowment effect }}+\underbrace{\frac{a}{a+b} \frac{\bar{q}_{2}}{p_{1}}}_{\text {constant-ratio effect }} \geq 0 \\
& {[=\underbrace{\frac{a b}{(a+b)^{2}} \frac{p_{1} \bar{q}_{1}+p_{2} \bar{q}_{2}}{p_{1} p_{2}}}_{\text {cross-substitution effect } \oplus}+\underbrace{\frac{a}{(a+b)^{2}} \frac{-b p_{1} \bar{q}_{1}+a p_{2} \bar{q}_{2}}{p_{1} p_{2}}}_{\text {income effect }}]}
\end{aligned}
$$

where $y=p_{1} \bar{q}_{1}+p_{2} \bar{q}_{2}$ and the last line in big brackets is due to the Slutsky equation (21). The cross-substitution effect and the income effect just cancel out as in (16). And so do the first and the second endowment effects. Thus the ratio effect vanishes and the cross-price effect is governed by the constant-ratio effect which is greater than or equal to zero.

As for the CES utility function (5), (22) is written as follows:

$$
\begin{aligned}
& \underbrace{\frac{(\sigma-1) c p_{1} \bar{q}_{1}+(\sigma c+1) p_{2} \bar{q}_{2}}{(1+c)^{2}} \frac{1}{p_{1} p_{2}}}_{\text {cross-price effect }}=\underbrace{\frac{(\sigma-1) c}{(1+c)^{2}} \frac{y}{p_{1} p_{2}}}_{\text {ratio effect }}+\underbrace{\frac{1}{1+c} \frac{\bar{q}_{2}}{p_{1}}}_{\text {constant-ratio effect }} \\
& =\underbrace{\frac{\sigma c}{(1+c)^{2}} \frac{y}{p_{1} p_{2}}}_{\text {cross-substitution effect } \oplus} \underbrace{-\frac{c}{(1+c)^{2}} \frac{y}{p_{1} p_{2}}}_{\text {income effect } \ominus} \underbrace{-\frac{c}{(1+c)^{2}} \frac{\bar{q}_{2}}{p_{1}}}_{\text {first endowment effect }} \\
& +\underbrace{\frac{c}{(1+c)^{2}} \frac{\bar{q}_{2}}{p_{1}}}_{\text {second endowment effect }}+\underbrace{\frac{1}{1+c} \frac{\bar{q}_{2}}{p_{1}}}_{\text {constant-ratio effect }} \gtreqless 0 \\
& {[\underbrace{\frac{\sigma c}{(1+c)^{2}} \frac{p_{1} \bar{q}_{1}+p_{2} \bar{q}_{2}}{p_{1} p_{2}}}_{\text {cross-substitution effect } \oplus}+\underbrace{\frac{1}{(1+c)^{2}} \frac{-c p_{1} \bar{q}_{1}+p_{2} \bar{q}_{2}}{p_{1} p_{2}}}_{\text {income effect }}]}
\end{aligned}
$$

where $y=p_{1} \bar{q}_{1}+p_{2} \bar{q}_{2}$ and the last line in brackets is the Slutsky decomposition for the cross-price effect (21). Since the first and the second endowment effects cancel out exactly, the ratio effect takes the same form as that in (17). The sign of the cross-price effect is indeterminate, but it is positive if $\sigma>1$. 


\section{Conclusion}

For a long time economists have made it a rule to decompose the price effect into two effects (the substitution effect and the income effect). They still believe that it is the only way to decompose the price effect even in the n-good case. This paper showed that the price effect can be decomposed into three effects (the substitution effect, the transfer effect, and the unit-elasticity effect) even in the two-good case. Since the sum of the transfer effect and the unit-elasticity effect is equal to the income effect, the new decomposition proposed in this paper can be regarded as a further decomposition of the traditional Slutsky decomposition.

This paper emphasized the usefulness of a new decomposition into the ratio effect and the unit-elasticity effect. The ratio effect consists of the two effects with opposite signs (the substitution effect and the transfer effect). If the two effects almost cancel out, the price effect is governed by the unit-elasticity effect. Especially if they cancel out exactly, the price elasticity of demand becomes one, which means that the demand function is derived from the Cobb-Douglas-type utility function.

The above result gives economists a new answer to the question of why the price elasticity for the demand function derived from the Cobb-Douglas is unity. A conventional answer would be, rather tautologically, "because a utility function which leads to a demand function with unitary price elasticity is the Cobb-Douglas." But, as the new equation shows clearly, any utility function is subject to the unit-elasticity effect. No good can escape from such an effect when its own price goes up. It is the canceling out of the substitution effect and the transfer effect that makes the price elasticity of demand just one. This is the answer the new equation can provide.

As far as a normal good is analyzed, the unit-elasticity effect always outweighs the ratio effect, which implies a downward sloping demand curve. This situation still holds in the case of an inferior good with a relatively small transfer effect. The new equation is able to deal with the case of a Giffen good, too, in which case a positive ratio effect is so large that the price effect becomes positive and the corresponding demand curve is upward sloping. Thus, a conventional rationale for the existence of a Giffen good is the dominance of a positive income effect over a negative substitution effect, whereas the rationale found by the new equation is an extraordinarily large transfer effect which means a great sacrifice of the other good. Furthermore, the new equation can be used for the consideration of the cross-price effect and the analysis of a case where a consumer holds initial endowments.

Finally I never intend to argue that the new decomposition is superior to the Slutsky decomposition. The new equation helps to see the world which cannot be seen by the Slutsky equation alone and vice versa. They are complements.

\section{Appendix 1: Conditions for Utility Maximization in Problem II}

Substituting $q_{1}=\theta y / p_{1}$ and $q_{2}=(1-\theta) y / p_{2}$ into utility function (1) yields

$$
v=u\left(\frac{\theta y}{p_{1}}, \frac{(1-\theta) y}{p_{2}}\right) .
$$


A consumer is in a position to maximize $v$ by adjusting the ratio $\theta$ only. Differentiate (24) with respect to $\theta$ once and twice. Then,

$$
\begin{aligned}
\frac{d v}{d \theta} & =u_{1} \frac{y}{p_{1}}-u_{2} \frac{y}{p_{2}} \\
\frac{d^{2} v}{d \theta^{2}} & =u_{11}\left(\frac{y}{p_{1}}\right)^{2}-2 u_{12} \frac{y}{p_{1}} \frac{y}{p_{2}}+u_{22}\left(\frac{y}{p_{2}}\right)^{2} .
\end{aligned}
$$

Putting $d v / d \theta=0$ gives the first-order condition

$$
\frac{u_{1}}{u_{2}}=\frac{p_{1}}{p_{2}}
$$

(25) assures that $d^{2} v / d \theta^{2}$ takes a negative value because

$$
\begin{aligned}
\frac{d^{2} v}{d \theta^{2}} & =\left(\frac{y}{p_{1}}\right)^{2}\left[u_{11}-2 u_{12} \frac{p_{1}}{p_{2}}+u_{22}\left(\frac{p_{1}}{p_{2}}\right)^{2}\right] \\
& =\left(\frac{y}{p_{1}}\right)^{2}\left[u_{11}-2 u_{12} \frac{u_{1}}{u_{2}}+u_{22}\left(\frac{u_{1}}{u_{2}}\right)^{2}\right] \\
& =\left(\frac{y}{p_{1}}\right)^{2} \frac{u_{2}^{2} u_{11}-2 u_{1} u_{2} u_{12}+u_{1}^{2} u_{22}}{u_{2}^{2}} \\
& =-\left(\frac{y}{p_{1}}\right)^{2} \frac{|U|}{u_{2}^{2}}<0 .
\end{aligned}
$$

Therefore, the second-order condition is also satisfied. ${ }^{23}$

\section{Appendix 2: Direct Method of Calculating the Ratio Effect (8)}

For the calculation of the ratio effect (8) it is necessary to notice that the first-order condition (25) always works. Thus the ratio $\theta$ can be written as

$$
\begin{aligned}
\theta & =\frac{p_{1} q_{1}}{y} \\
& =\frac{\frac{p_{1}}{p_{2}} q_{1}}{\frac{p_{1}}{p_{2}} q_{1}+q_{2}}
\end{aligned}
$$

23 The extension of this two-good case to an $n$-good case is easy. When there are $n$ goods, (24) becomes

$$
v=u\left(\frac{\theta_{1} y}{p_{1}}, \frac{\theta_{2} y}{p_{2}}, \ldots, \frac{\left[1-\left(\theta_{1}+\theta_{2}+\ldots+\theta_{n-1}\right)\right] y}{p_{n}}\right),
$$

where $\theta_{i}$ represents the ratio of income $y$ that goes to the purchase of good $i, i=1,2, \ldots, n-1$. The maximization conditions in this case are $d v / d \theta_{i}=0$ and $d^{2} v / d \theta_{i}^{2}<0, i=1,2, \ldots, n-1$. 


$$
\begin{aligned}
& =\frac{\frac{u_{1}}{u_{2}} q_{1}}{\frac{u_{1}}{u_{2}} q_{1}+q_{2}} \\
& =\frac{u_{1} q_{1}}{u_{1} q_{1}+u_{2} q_{2}} .
\end{aligned}
$$

(26) also means

$$
u_{1} q_{1}+u_{2} q_{2}=u_{1} \frac{y}{p_{1}} .
$$

Differentiate (26) w.r.t. $p_{1}$. Then,

$$
\frac{d \theta}{d p_{1}}=\frac{\frac{d\left(u_{1} q_{1}\right)}{d p_{1}} u_{2} q_{2}-\frac{d\left(u_{2} q_{2}\right)}{d p_{1}} u_{1} q_{1}}{\left(u_{1} q_{1}+u_{2} q_{2}\right)^{2}} .
$$

Multiplying both sides of the above equation by $\left(u_{1} q_{1}+u_{2} q_{2}\right)^{2}$ gives

$$
\left(u_{1} q_{1}+u_{2} q_{2}\right)^{2} \frac{d \theta}{d p_{1}}=\frac{d\left(u_{1} q_{1}\right)}{d p_{1}} u_{2} q_{2}-\frac{d\left(u_{2} q_{2}\right)}{d p_{1}} u_{1} q_{1} .
$$

Using (27) it can be rewritten as

$$
\left(u_{1} \frac{y}{p_{1}}\right)^{2} \frac{d \theta}{d p_{1}}=\frac{d\left(u_{1} q_{1}\right)}{d p_{1}} u_{2} q_{2}-\frac{d\left(u_{2} q_{2}\right)}{d p_{1}} u_{1} q_{1} .
$$

By the way, it can easily be checked that the following calculations are correct:

$$
\begin{aligned}
& \frac{d\left(u_{1} q_{1}\right)}{d p_{1}}=u_{11}\left(\frac{\frac{d \theta}{d p_{1}} y}{p_{1}}-\frac{q_{1}}{p_{1}}\right) q_{1}-u_{12} \frac{\frac{d \theta}{d p_{1}} y}{p_{2}} q_{1}+u_{1}\left(\frac{\frac{d \theta}{d p_{1}} y}{p_{1}}-\frac{q_{1}}{p_{1}}\right), \\
& \frac{d\left(u_{2} q_{2}\right)}{d p_{1}}=u_{12}\left(\frac{\frac{d \theta}{d p_{1}} y}{p_{1}}-\frac{q_{1}}{p_{1}}\right) q_{2}-u_{22} \frac{\frac{d \theta}{d p_{1}} y}{p_{2}} q_{2}-u_{2} \frac{\frac{d \theta}{d p_{1}} y}{p_{2}} .
\end{aligned}
$$

So substitute (29) and (30) into (28). Then,

$$
\begin{aligned}
\left(u_{1} \frac{y}{p_{1}}\right)^{2} \frac{d \theta}{d p_{1}}= & {\left[u_{11}\left(\frac{\frac{d \theta}{d p_{1}} y}{p_{1}}-\frac{q_{1}}{p_{1}}\right) q_{1}-u_{12} \frac{p_{1}}{p_{2}} \frac{\frac{d \theta}{d p_{1}} y}{p_{1}} q_{1}+u_{1}\left(\frac{\frac{d \theta}{d p_{1}} y}{p_{1}}-\frac{q_{1}}{p_{1}}\right)\right] u_{2} q_{2} } \\
& -\left[u_{12}\left(\frac{\frac{d \theta}{d p_{1}} y}{p_{1}}-\frac{q_{1}}{p_{1}}\right) q_{2}-u_{22} \frac{p_{1}}{p_{2}} \frac{\frac{d \theta}{d p_{1}} y}{p_{1}} q_{2}-u_{2} \frac{p_{1}}{p_{2}} \frac{\frac{d \theta}{d p_{1}} y}{p_{1}}\right] u_{1} q_{1} .
\end{aligned}
$$

In order to reach a desired result a few more steps are needed. That is,

$$
\begin{aligned}
& \left(u_{1}^{2} \frac{y}{p_{1}}-u_{2} u_{11} q_{1} q_{2}+u_{1} u_{12} q_{1} q_{2}-u_{1} u_{2} q_{2}+u_{1} u_{12} q_{1} q_{2}-\frac{u_{1}^{2}}{u_{2}} u_{22} q_{1} q_{2}-u_{1}^{2} q_{1}\right) \frac{\frac{d \theta}{d p_{1}} y}{p_{1}} \\
& =\left[-\frac{1}{p_{1}} u_{1} u_{2}+\frac{q_{1}}{p_{1}}\left(u_{1} u_{12}-u_{2} u_{11}\right)\right] q_{1} q_{2},
\end{aligned}
$$




$$
\begin{aligned}
& \left(u_{1}^{2} \frac{u_{1} q_{1}+u_{2} q_{2}}{u_{1}}-u_{2} u_{11} q_{1} q_{2}+2 u_{1} u_{12} q_{1} q_{2}-u_{1} u_{2} q_{2}-\frac{u_{1}^{2}}{u_{2}} u_{22} q_{1} q_{2}-u_{1}^{2} q_{1}\right) \frac{d \theta}{d p_{1}} y \\
& =\left[-\frac{1}{p_{1}} u_{1} u_{2}+\frac{q_{1}}{p_{1}}\left(u_{1} u_{12}-u_{2} u_{11}\right)\right] q_{1} q_{2}, \\
& \left(-u_{2} u_{11} q_{1} q_{2}+2 u_{1} u_{12} q_{1} q_{2}-\frac{u_{1}^{2}}{u_{2}} u_{22} q_{1} q_{2}\right) \frac{\frac{d \theta}{d p_{1}} y}{p_{1}} \\
& =\left[-\frac{1}{p_{1}} u_{1} u_{2}+\frac{q_{1}}{p_{1}}\left(u_{1} u_{12}-u_{2} u_{11}\right)\right] q_{1} q_{2},
\end{aligned}
$$

and finally

$$
\left(-u_{2}^{2} u_{11}+2 u_{1} u_{2} u_{12}-u_{1}^{2} u_{22}\right) \frac{\frac{d \theta}{d p_{1}} y}{p_{1}}=-\frac{u_{1}}{p_{1}} u_{2}^{2}+\frac{q_{1}}{p_{1}}\left(u_{1} u_{2} u_{12}-u_{2}^{2} u_{11}\right) .
$$

Dividing both sides of the last equation by the bordered Hessian $|U|$ gives the ratio effect (8)

$$
\frac{\frac{d \theta}{d p_{1}} y}{p_{1}}=-\frac{u_{1}}{p_{1}} \frac{u_{2}^{2}}{|U|}+\frac{q_{1}}{p_{1}} \frac{u_{1} u_{2} u_{12}-u_{2}^{2} u_{11}}{|U|}
$$

\section{Appendix 3: Direct Method of Calculating the Ratio Effect (15) in the Cross-Price Effect}

The derivation of (15) is similar to that of (8) shown in "Appendix 2". Differentiating (26) w.r.t. $p_{2}$ and considering (27) yields

$$
\left(u_{1} \frac{y}{p_{1}}\right)^{2} \frac{d \theta}{d p_{2}}=\frac{d\left(u_{1} q_{1}\right)}{d p_{2}} u_{2} q_{2}-\frac{d\left(u_{2} q_{2}\right)}{d p_{2}} u_{1} q_{1} .
$$

Notice here that the counterpart of Eq. (7) also obtains, that is,

$$
\left.\frac{d q_{2}}{d p_{2}}\right|_{p_{1}, y=\mathrm{const}}=-\frac{\frac{d \theta}{d p_{2}} y}{p_{2}}-\frac{q_{2}}{p_{2}} .
$$

Keeping this in mind, one can obtain the following relations at once:

$$
\begin{aligned}
& \frac{d\left(u_{1} q_{1}\right)}{d p_{2}}=u_{11} \frac{\frac{d \theta}{d p_{2}} y}{p_{1}} q_{1}+u_{12}\left(-\frac{\frac{d \theta}{d p_{2}} y}{p_{2}}-\frac{q_{2}}{p_{2}}\right) q_{1}+u_{1} \frac{\frac{d \theta}{d p_{2}} y}{p_{1}}, \\
& \frac{d\left(u_{2} q_{2}\right)}{d p_{2}}=u_{12} \frac{\frac{d \theta}{d p_{2}} y}{p_{1}} q_{2}+u_{22}\left(-\frac{\frac{d \theta}{d p_{2}} y}{p_{2}}-\frac{q_{2}}{p_{2}}\right) q_{2}+u_{2}\left(-\frac{\frac{d \theta}{d p_{2}} y}{p_{2}}-\frac{q_{2}}{p_{2}}\right) .
\end{aligned}
$$


Substituting (32) and (33) into (31) and arranging the resulting terms yields

$$
\left(-u_{2}^{2} u_{11}+2 u_{1} u_{2} u_{12}-u_{1}^{2} u_{22}\right) \frac{\frac{d \theta}{d p_{2}} y}{p_{1}}=\frac{1}{p_{2}} u_{1} u_{2}^{2}-q_{2} \frac{u_{2}}{p_{2}}\left(u_{2} u_{12}-u_{1} u_{22}\right) .
$$

Finally divide both sides of the above equation by the bordered Hessian $|U|$. Then the desired result, i.e., (15), follows:

$$
\frac{\frac{d \theta}{d p_{2}} y}{p_{1}}=\frac{u_{2}}{p_{2}} \frac{u_{1} u_{2}}{|U|}-q_{2} \frac{u_{2}}{p_{2}} \frac{u_{2} u_{12}-u_{1} u_{22}}{|U|} .
$$

\section{References}

Allen RGD (1936) Professor Slutsky's theory of consumers' choice. Rev Econ Stud 3:120-129

Arrow KJ, Chenery HB, Minhas BS, Solow RM (1961) Capital-labor sustitution and economic efficiency. Rev Econ Stat 43:225-250

Battalio RC, Kagel JH, Kogut CA (1991) Experimental confirmation of the existence of a Giffen good. Am Econ Rev 81:961-970

Cobb CW, Douglas PH (1928) A theory of production. Am Econ Rev 18(Supplement):139-165

Dooley PC (1983) Slutsky's equation is Pareto's solution. History Polit Econ 15:513-517

Doi J, Iwasa K, Shimomura K (2009) Giffen behavior independent of the wealth level. Econ Theory 41:247267

Douglas PH (1967) Comments on the Cobb-Douglas production function. In: Brown M (ed) The theory and empirical analysis of production. National Bureau of Economic Research, New York, pp 15-22

Hall RE (1988) Intertemporal substitution in consumption. J Polit Econ 96:339-357

Henderson JM, Quandt RE (1980) Microeconomic theory: a mathematical approach, 3rd edn. McGraw-Hill, New York

Hicks JR (1939) Value and capital: an inquiry into some fundamental principles of economic theory. Clarendon Press, Oxford

Hicks JR, Allen RGD (1934a) A reconsideration of the theory of value, Part I. Economica 1:52-76

Hicks JR, Allen RGD (1934b) A reconsideration of the theory of value, Part II. Economica 1:196-219

Jensen RT, Miller NH (2008) Giffen behavior and subsistence consumption. Am Econ Rev 98:1553-1577

Leibenstein H (1950) Bandwagon, Snob, and Veblen effects in the theory of consumers' demand. Q J Econ 64:183-207

Moffatt PG (2002) Is Giffen behaviour compatible with the axioms of consumer theory? J Math Econ $37: 259-267$

Montesano A (1985) The ordinal utility under uncertainty and the measure of risk aversion in terms of preferences. Theory Decis 18:73-85

Pareto V (1892-93) Considerazioni sui Principii Fondamentali dell'Economia Politica Pura. G Econ 4:389_ 420; 4:485-512; 5:119-157; 6:1-37; 7:279-321. Translated as Roberto Marchionatti and Fiorenzo Mornati (eds) (2007) Considerations on the fundamental principles of pure political economy. Routledge, London

Schultz H (1935) Interrelations of demand, price, and income. J Polit Econ 43:433-481

Silberberg E, Walker DA (1984) A modern analysis of Giffen's paradox. Int Econ Rev 25:687-694

Slutsky EE (1915) Sulla Teoria del Bilancio del Consumatore. G Econ Riv Stat 51:1-26. Translated as On the theory of the budget of the consumer. In: Stigler George J, Boulding Kenneth E (eds) (1952)

Readings in price theory. Richard D. Irwin, Chicago, pp 27-56

Solow RM (2005) Reflections on growth theory. In: Aghion P, Durlauf SN (eds) Handbook of economic growth, 1A. Elsevier, Amsterdam, pp 3-10

Spiegel U (1994) The case of a "Giffen Good”. J Econ Educ 25:138-147

Stigler GJ (1947) Notes on the history of the Giffen paradox. J Polit Econ 55:152-156

Stigler GJ (1987) The theory of price, 4th edn. Macmillan, New York

Stiglitz JE, Walsh CE (2006) Economics, 4th edn. W. W. Norton, New York 
Vandermeulen DC (1972) Upward sloping demand curves without the Giffen paradox. Am Econ Rev 62:453-458

Whitehead JA (2010) Microeconomics: a global text. Routledge, London

Wold Herman OA (1948) On Giffen's paradox, Nordisk Tidsskrift for Teknisk Ø konomi, vol 12, pp 283290. Reprinted in Heijman W, von Mouche P (eds) (2012) New insights into the theory of Giffen goods. Springer, Berlin, pp 89-96 